\title{
LAPTH
}

Laboratoire d'Annecy-le-Vieux de Physique Théorique

\section{Vertex operators for quantum groups and application to integrable systems}

\author{
E. Ragoucy' \\ Laboratoire de Physique Théorique LAPTH币 \\ LAPP, BP 110, F-74941 Annecy-le-Vieux Cedex, France.
}

\begin{abstract}
Starting with any $R$-matrix with spectral parameter, obeying the YangBaxter equation and a unitarity condition, we construct the corresponding infinite dimensional quantum group $\mathcal{U}_{R}$ in term of a deformed oscillators algebra $\mathcal{A}_{R}$. The realization we present is an infinite series, very similar to a vertex operator.

Then, considering the integrable hierarchy naturally associated to $\mathcal{A}_{R}$, we show that $\mathcal{U}_{R}$ provides its integrals of motion. The construction can be applied to any infinite dimensional quantum group, e.g. Yangians or elliptic quantum groups.

Taking as an example the $R$-matrix of $Y(N)$, the Yangian based on $g l(N)$, we recover by this construction the nonlinear Schrödinger equation and its $Y(N)$ symmetry.
\end{abstract}

mathQA/0108207

LAPTH-859/01

July 01

*ragoucy@lapp.in2p3.fr

†UMR 5108 du CNRS, associée à l’Université de Savoie. 


\section{Introduction}

The aim of this paper is to present a general construction of infinite dimensional quantum groups as explicit integrals of motions of integrable systems. The construction relies only on the existence of an evaluated $R$-matrix (with spectral parameter) which obeys the unitarity condition. Thus, it can be applied to any infinite dimensional quantum group.

To the $R$-matrix, one can associate a ZF algebra $\mathcal{A}_{R}$ [1], which, in a Fock space representation provides the asymptotic states of the model. The quantum group is then constructed as an infinite series in the ZF generators, and shown to commute with the Hamiltonian of the hierarchy. Thus, it generates the integrals of motion of the hierarchy. Moreover, since there is a natural action of the quantum group on the $\mathcal{A}_{R}$ generators, its action on asymptotic states of the system is easily deduced.

Taking as an example the $R$-matrix of $Y(N)$, the Yangian based on $g l(N)$, we recover by this construction the nonlinear Schrödinger equation and its $Y(N)$ symmetry [2, 3]. It is thus very natural to believe that the other integrable systems known in the literature can be treated with the present approach.

The paper is organized as follows. In the section 2, we introduce the different definitions and properties we will need. From these notions, we construct, in section 3, a quantum group $\mathcal{U}_{R}$ from the deformed oscillator algebra $\mathcal{A}_{R}$. We consider in section 0 the hierarchy associated to $\mathcal{A}_{R}$ and show that $\mathcal{U}_{R}$ generates integrals of motion. Then, its Fock space representation is studied in section 5. Section 6 deals with three examples: the nonlinear Schrödinger equation with its Yangian symmetry (case of additive spectral parameter $R$-matrix), and $\mathcal{U}_{q}\left(\widehat{g l_{2}}\right)$ and $\mathcal{A}_{q, p}\left(g l_{2}\right)$ (case of multiplicative $R$-matrix). Finally, we conclude in section 7 .

\section{Definitions and first properties}

\subsection{Z.F. algebras}

We start with an $R$-matrix satisfying the Yang-Baxter equation with spectral parameter:

$$
R_{12}\left(k_{1}, k_{2}\right) R_{13}\left(k_{1}, k_{3}\right) R_{23}\left(k_{2}, k_{3}\right)=R_{23}\left(k_{2}, k_{3}\right) R_{13}\left(k_{1}, k_{3}\right) R_{12}\left(k_{1}, k_{2}\right)
$$

and the unitarity condition

$$
R_{12}\left(k_{1}, k_{2}\right) R_{21}\left(k_{2}, k_{1}\right)=\mathbb{I} \otimes \mathbb{I}
$$

$R$ is an $N^{2} \times N^{2}$ matrix. Here and below we will denote for briefness

$$
R_{12} \equiv R_{12}\left(k_{1}, k_{2}\right)
$$


but let us stress that the $R$-matrix we consider are defined with spectral parameter. Note also that both the usual additive and multiplicative cases for the $R$-matrix, where $R\left(k_{1}, k_{2}\right)$ stands for $R\left(k_{1}-k_{2}\right)$ and $R\left(k_{1} / k_{2}\right)$ respectively, are included in our formalism.

\section{Definition 2.1 (ZF algebra $\mathcal{A}_{R}$ )}

To each R-matrix obeying (2.1) and (2.9), one can associate a Zamolodchikov-Faddeev (ZF) algebra $\mathcal{A}_{R}$ [1], with generators $a_{i}(k)$ and $a_{i}^{\dagger}(k)(i=1, \ldots, N)$ and exchange relations:

$$
\begin{aligned}
& a_{1} a_{2}=R_{21} a_{2} a_{1} \\
& a_{1}^{\dagger} a_{2}^{\dagger}=a_{2}^{\dagger} a_{1}^{\dagger} R_{21} \\
& a_{1} a_{2}^{\dagger}=a_{2}^{\dagger} R_{12} a_{1}+\delta_{12}
\end{aligned}
$$

We have used the notations

$$
\begin{aligned}
& a_{1}=\sum_{i=1}^{N} a_{i}\left(k_{1}\right) e_{i} \otimes \mathbb{I} \quad, \quad a_{2}=\sum_{i=1}^{N} a_{i}\left(k_{2}\right) \mathbb{I} \otimes e_{i} \\
& a_{1}^{\dagger}=\sum_{i=1}^{N} a_{i}^{\dagger}\left(k_{1}\right) e_{i}^{\dagger} \otimes \mathbb{I} \quad, \quad a_{2}^{\dagger}=\sum_{i=1}^{N} a_{i}^{\dagger}\left(k_{2}\right) \mathbb{I} \otimes e_{i}^{\dagger} \\
& \delta_{12}=\delta\left(k_{1}-k_{2}\right) \sum_{i=1}^{N} e_{i} \otimes e_{i}^{\dagger} \quad, \quad e_{i}^{\dagger}=(0, \ldots, 0, \stackrel{i}{1}, 0, \ldots, 0) \quad, \quad e_{i}^{\dagger} \cdot e_{j}=\delta_{i j}
\end{aligned}
$$

where $\cdot$ stands for vectors scalar product.

Let us remark that, in the same way the Yang-Baxter equation ensures the associativity of the product in $\mathcal{A}_{R}$, the unitarity condition can be interpreted as a consistency condition for the $\mathcal{A}_{R}$ algebra. Indeed, starting with (2.4), exchanging the auxiliary spaces $1 \leftrightarrow 2$ and the spectral parameters $k_{1} \leftrightarrow k_{2}$, and multiplying by $\left(R_{12}\right)^{-1}$ one gets

$$
a_{1} a_{2}=\left(R_{12}\right)^{-1} a_{2} a_{1}
$$

Comparing this last relation with (2.4), we recover the unitarity condition.

Above and in the following, we will loosely write $a_{1} \in \mathcal{A}_{R}$.

\section{Property 2.2 (Adjoint anti-automorphism)}

$$
\text { Let } \dagger \text { be the operation defined by } \begin{cases}\mathcal{A}_{R} & \rightarrow \mathcal{A}_{R} \\ a(k) & \mapsto a^{\dagger}(k) \\ a^{\dagger}(k) & \mapsto a(k) \\ R_{12}\left(k_{1}, k_{2}\right) & \mapsto R_{21}\left(k_{2}, k_{1}\right)\end{cases}
$$

and $(x y)^{\dagger}=y^{\dagger} x^{\dagger} \forall x, y \in \mathcal{A}_{R}$. Then $\dagger$ is an automorphism of the $\mathcal{A}_{R}$ algebra, and we can make the identifications $(a)^{\dagger} \equiv a^{\dagger}$ and $\left(a^{\dagger}\right)^{\dagger} \equiv a$. 
Proof: Direct calculation. For instance:

$$
\begin{aligned}
\left(a_{1} a_{2}\right)^{\dagger} & =\left(a_{2}\right)^{\dagger}\left(a_{1}\right)^{\dagger} \\
& =\left(a_{1}\right)^{\dagger}\left(a_{2}\right)^{\dagger}\left(R_{21}\right)^{\dagger}=\left(a_{1}\right)^{\dagger}\left(a_{2}\right)^{\dagger} R_{12}
\end{aligned}
$$

After the exchange $1 \leftrightarrow 2$, one recovers 2.5$)$ :

$$
\left(a_{1}\right)^{\dagger}\left(a_{2}\right)^{\dagger}=\left(a_{2}\right)^{\dagger}\left(a_{1}\right)^{\dagger} R_{21}
$$

The other relations are obtained in the same way, once one remarks $\left(\delta_{21}\right)^{\dagger}=\delta_{12}$.

\section{$2.2 \quad$ Vertex operators}

\section{Definition 2.3 (Vertex operators)}

The vertex operators $T^{i j}(k),(i, j=1 \ldots, N)$ associated to the algebra $\mathcal{A}_{R}$ are defined by $T(k) \equiv T^{i j}(k) E_{i j} \in \mathcal{A}_{R} \otimes \mathbb{C}^{N^{2}}$ where

$$
T\left(k_{\infty}\right)=\mathbb{I}+\sum_{n=1}^{\infty} \frac{(-1)^{n}}{n !} a_{n \ldots 1}^{\dagger} T_{\infty 1 \ldots n}^{(n)} a_{1 \ldots n}
$$

with

$$
\begin{aligned}
a_{n \ldots 1}^{\dagger} & =a_{\alpha_{n}}^{\dagger}\left(k_{n}\right) \ldots a_{\alpha_{1}}^{\dagger}\left(k_{1}\right) \\
a_{1 \ldots n} & =a_{\beta_{1}}\left(k_{1}\right) \ldots a_{\beta_{n}}\left(k_{n}\right) \\
T_{\infty 1 \ldots n}^{(n)} & =T_{\infty, \alpha_{1}, \beta_{1}, \ldots \alpha_{n}, \beta_{n}}^{(n)}\left(k_{\infty}, k_{1}, \ldots, k_{n}\right) \in\left(\mathbb{C}^{\otimes N^{2}}\right)^{\otimes(n+1)}\left(k_{\infty}, k_{1}, \ldots, k_{n}\right)
\end{aligned}
$$

In (2.10), there is an implicit summation on the indices $\alpha_{1}, \beta_{1}, \ldots, \alpha_{n}, \beta_{n}=1, \ldots, N$ and an integration over the spectral parameters $k_{1}, \ldots, k_{n}$.

For convenience, $\infty$ labels the auxiliary space associated to $T\left(k_{\infty}\right)$, and, as for the $R$-matrix, we will note $T_{\infty} \equiv T_{\infty}\left(k_{\infty}\right)$.

Let us stress that, in the notation (2.10), the auxiliary spaces $1, \ldots, n$ are "internal" in the sense that the indices corresponding to these spaces are summed and define scalars, not matrices, in these spaces. It is only the indices corresponding to the "external" auxiliary space $\infty$ which refers to the matrix labeling for $T$. For instance $a_{1}^{\dagger} T_{\infty 1}^{(1)} a_{1}$ stands for

$$
a_{1}^{\dagger} T_{\infty 1}^{(1)} a_{1}=\sum_{\alpha, \beta=1}^{N}\left(a_{1}^{\dagger} T_{\infty 1}^{(1)} a_{1}\right)_{\alpha, \beta} E_{\alpha, \beta}=\sum_{\alpha, \beta=1}^{N}\left(\sum_{\gamma, \mu=1}^{N} a_{\gamma}^{\dagger} T_{\alpha, \beta ; \gamma, \mu}^{(1)} a_{\mu}\right) E_{\alpha, \beta}
$$

so that we could have written $a_{2}^{\dagger} T_{\infty 2}^{(1)} a_{2}$ as well: $1, \ldots, n$ are dummy space indices. 
Remark 1 The series (2.10) is very similar to a normal ordered (in $a$ and $a^{\dagger}$ ) exponential

$$
V\left(k_{\infty}\right)=: \exp \left(-a^{\dagger} M a\right):
$$

whence the denomination vertex operator used here to denote it.

\section{Property $2.4\left(\mathcal{S}_{n}\right.$-covariance of the vertex operators)}

The vertex operators coefficients $T_{\infty 1 \ldots n}^{(n)}$ are covariant under the action of the permutation group $\mathcal{S}_{n}$.

More precisely, for $\sigma \in \mathcal{S}_{n}$, one has:

$$
T_{\infty \sigma(1) \ldots \sigma(n)}^{(n)}=\mathcal{R}_{\sigma}^{1 \ldots n} T_{\infty 1 \ldots n}^{(n)}\left(\mathcal{R}_{\sigma}^{1 \ldots n}\right)^{-1}
$$

where $\mathcal{R}_{\sigma}^{1 \ldots n}$ is the product of $R$-matrices defined by $a_{\sigma(1) \ldots \sigma(n)}=\mathcal{R}_{\sigma}^{1 \ldots n} a_{1 \ldots n}$.

$\underline{\text { Proof: }}$ Starting from the term $X_{n}=a_{n \ldots 1}^{\dagger} T_{\infty 1 \ldots n}^{(n)} a_{1 \ldots n}$ and relabeling the auxiliary spaces $i \rightarrow \sigma(i)$ (and also the spectral parameters), one gets

$$
X_{n}=a_{\sigma(n) \ldots \sigma(1)}^{\dagger} T_{\infty \sigma(1) \ldots \sigma(n)}^{(n)} a_{\sigma(1) \ldots \sigma(n)}
$$

Then, from the exchange properties of the $a$ 's and $a^{\dagger}$ 's and the property 2.2, one has:

$$
a_{\sigma(n) \ldots \sigma(1)}^{\dagger}=a_{n \ldots 1}^{\dagger}\left(\mathcal{R}_{\sigma}^{1 \ldots n}\right)^{-1} \quad \text { and } \quad a_{\sigma(1) \ldots \sigma(n)}=\mathcal{R}_{\sigma}^{1 \ldots n} a_{1 \ldots n}
$$

which leads to the formula (2.15).

As an example, if $\sigma$ is just the transposition $i \leftrightarrow i+1$, one gets $\mathcal{R}_{\sigma}^{1 \ldots n}=R_{i, i+1}$ and the formula

$$
T_{\infty 1 \ldots i-1, i+1, i, i+2 \ldots n}^{(n)}=R_{i, i+1} T_{\infty 1 \ldots n}^{(n)} R_{i+1, i}
$$

Property 2.5 The matrices $\mathcal{R}_{\sigma}^{1 \ldots n}, \sigma \in \mathcal{S}_{n}$, defined by

$$
a_{\sigma(1) \ldots \sigma(n)}=\mathcal{R}_{\sigma}^{1 \ldots n} a_{1 \ldots n}
$$

obey to

$$
\mathcal{R}_{\sigma}^{\mu(1) \ldots \mu(n)} \mathcal{R}_{\mu}^{1 \ldots n}=\mathcal{R}_{\sigma o \mu}^{1 \ldots n} \quad \text { so that } \quad\left(\mathcal{R}_{\sigma}^{1 \ldots n}\right)^{-1}=\mathcal{R}_{\sigma^{-1}}^{\sigma(1) \ldots \sigma(n)}
$$

From any matrix $M_{1 \ldots n} \in\left(\mathbb{C}^{N^{2}}\right)^{\otimes n}$, one can construct a $\mathcal{S}_{n}$-covariant one by

$$
\widetilde{M}_{1 \ldots n}=\frac{1}{n !} \sum_{\sigma \in \mathcal{S}_{n}}\left(\mathcal{R}_{\sigma}^{1 \ldots n}\right)^{-1} M_{\sigma(1) \ldots \sigma(n)} \mathcal{R}_{\sigma}^{1 \ldots n}
$$


Proof: The first formula is proved by direct calculation:

$$
a_{\sigma \circ \mu(1) \ldots \sigma \circ \mu(n)}=\mathcal{R}_{\sigma \circ \mu}^{1 \ldots n} a_{1 \ldots n}=\mathcal{R}_{\sigma}^{\mu(1) \ldots \mu(n)} a_{\mu(1) \ldots \mu(n)}=\mathcal{R}_{\sigma}^{\mu(1) \ldots \mu(n)} \mathcal{R}_{\mu}^{1 \ldots n} a_{1 \ldots n}
$$

Now, for the last formula, one has (for any $\mu \in \mathcal{S}_{n}$ ):

$$
\begin{aligned}
\widetilde{M}_{\mu(1) \ldots \mu(n)} & =\frac{1}{n !} \sum_{\sigma \in \mathcal{S}_{n}}\left(\mathcal{R}_{\sigma}^{\mu(1) \ldots \mu(n)}\right)^{-1} M_{\sigma \circ \mu(1) \ldots \sigma \circ \mu(n)} \mathcal{R}_{\sigma}^{\mu(1) \ldots \mu(n)} \\
& =\frac{1}{n !} \sum_{\sigma^{\prime} \in \mathcal{S}_{n}}\left(\mathcal{R}_{\sigma^{\prime} \circ \mu^{-1}}^{\mu(1) \ldots \mu(n)}\right)^{-1} M_{\sigma^{\prime}(1) \ldots \sigma^{\prime}(n)} \mathcal{R}_{\sigma^{\prime} \circ \mu^{-1}}^{\mu(1) \ldots \mu(n)}
\end{aligned}
$$

where in the last expression, we have made the change of variable $\sigma^{\prime}=\sigma \circ \mu$. Now, using (2.20), one gets $\mathcal{R}_{\sigma^{\prime} \circ \mu^{-1}}^{\mu(1) \ldots(n)}=\mathcal{R}_{\sigma^{\prime}}^{1 \ldots n}\left(\mathcal{R}_{\mu}^{1 \ldots n}\right)^{-1}$, and $\left(\mathcal{R}_{\sigma^{\prime} \circ \mu^{-1}}^{\mu(1) \ldots \mu(n)}\right)^{-1}=\mathcal{R}_{\mu}^{1 \ldots n}\left(\mathcal{R}_{\sigma^{\prime}}^{1 \ldots n}\right)^{-1}$, so that $\widetilde{M}_{1 \ldots n}$ is $\mathcal{S}_{n \text {-covariant. }}$

Remark 2 Strictly speaking, one can start with vertex operators which does not obey the $\mathcal{S}_{n}$-covariance (2.15), but the relevant part in the vertex operator will be the covariant one, as given by (2.21).

\subsection{Well-bred operators}

\section{Definition 2.6 (well-bred operators)}

An operator $L$ is said well-bred (on $\mathcal{A}_{R}$ ) when it acts on a and $a^{\dagger}$ as

$$
L_{1} a_{2}=R_{21} a_{2} L_{1} \quad \text { and } \quad L_{1} a_{2}^{\dagger}=a_{2}^{\dagger} R_{12} L_{1}
$$

We give few properties of well-bred operators that will be useful in the following.

Lemma 2.7 Let $L$ be a well-bred operator, then $L^{\dagger}(k) L(k)$ is central in $\mathcal{A}_{R}$.

Proof: One applies the $\dagger$ automorphism to the relations (2.23). We get:

$$
a_{2}^{\dagger} L_{1}^{\dagger}=L_{1}^{\dagger} a_{2}^{\dagger} R_{12} \quad \text { and } \quad a_{2} L_{1}^{\dagger}=L_{1}^{\dagger} R_{21} a_{2}^{\dagger}
$$

Then a direct calculation shows that $L^{\dagger}(k) L(k)$ commutes with $a$ and $a^{\dagger}$. For instance

$$
L_{1}^{\dagger} L_{1} a_{2}^{\dagger}=L_{1}^{\dagger} a_{2}^{\dagger} R_{12} L_{1}=a_{2}^{\dagger} L_{1}^{\dagger} L_{1}
$$

*We call these operators "well-bred" because they act nicely (on $a$ and $a^{\dagger}$ ). 
Lemma 2.8 Let $L$ be a well-bred operator of $\mathcal{A}_{R}$. Then $c_{12}=L_{1}^{-1} L_{2}^{-1} R_{12} L_{1} L_{2}$ is central in $\mathcal{A}_{R}$. It satisfies $c_{12}^{-1}=c_{21}$.

Proof: Starting with (2.23), one gets

$$
L_{1} L_{2} a_{3}=R_{32} R_{31} a_{3} L_{1} L_{2}
$$

which can be rewritten (after exchange $1 \leftrightarrow 2$ ) as

$$
R_{31} R_{32} a_{3}=L_{2} L_{1} a_{3} L_{1}^{-1} L_{2}^{-1}
$$

Then

$$
\begin{aligned}
R_{12} L_{1} L_{2} a_{3} & =R_{12} R_{32} R_{31} a_{3} L_{1} L_{2}=R_{31} R_{32} R_{12} a_{3} L_{1} L_{2}=R_{31} R_{32} a_{3} R_{12} L_{1} L_{2} \\
& =L_{2} L_{1} a_{3} L_{1}^{-1} L_{2}^{-1} R_{12} L_{1} L_{2}
\end{aligned}
$$

So that, multiplying by $L_{1}^{-1} L_{2}^{-1}$, we obtain

$$
c_{12} a_{3}=a_{3} c_{12}
$$

Performing a similar calculation with $a_{3}^{\dagger}$, we get $c_{12} a_{3}^{\dagger}=a_{3}^{\dagger} c_{12}$.

The last equation is a direct consequence of the unitarity condition.

\section{Construction of well-bred vertex operators}

We first give a characterization of well-bred vertex operators:

Lemma 3.1 The vertex operators $T$ is well-bred if and only if $T_{\infty 1 \ldots n}^{(n)}$ obeys

$$
\begin{aligned}
& T_{\infty 0}^{(1)}=\mathbb{I}-R_{\infty 0} \quad \text { and for } \quad n \geq 1: \\
& (n+1)\left\{T_{\infty 1 \ldots n}^{(n)}-\left(\mathcal{R}_{0, n}\right)^{-1} R_{\infty 0} T_{\infty 1 \ldots n}^{(n)} \mathcal{R}_{0, n}\right\}=\sum_{i=1}^{n+1}\left(\mathcal{R}_{0, i-1}\right)^{-1} T_{\infty 1 \ldots n \mid i}^{(n+1)} \mathcal{R}_{0, i-1}
\end{aligned}
$$

where we have introduced

$$
\mathcal{R}_{0, n}=\overleftarrow{\prod_{a=1}^{n}} R_{0 a} \quad ; \quad T_{\infty 1 \ldots n \mid i}^{(n+1)}=T_{\infty 1 \ldots i-1,0, i \ldots n}^{(n+1)}(i \leq n) \quad \text { and } \quad T_{\infty 1 \ldots n \mid n+1}^{(n+1)}=T_{\infty 1 \ldots n 0}^{(n+1)}
$$


Proof: We prove the property by a direct calculation. We note $\widehat{T}_{\infty}=T_{\infty}-\mathbb{I}$ :

$$
\begin{aligned}
a_{0} \widehat{T}_{\infty}= & \sum_{n=1}^{\infty} \frac{(-1)^{n}}{n !}\left(a_{n}^{\dagger} R_{0 n} a_{0}+\delta_{0 n}\right) a_{n-1 \ldots 1}^{\dagger} T_{\infty 1 \ldots n}^{(n)} a_{1 \ldots n} \\
= & \sum_{n=1}^{\infty} \frac{(-1)^{n}}{n !}\left\{a_{n \ldots 1}^{\dagger} R_{0 n} \cdots R_{01} a_{0} T_{\infty 1 \ldots n}^{(n)} a_{1 \ldots n}\right. \\
& \left.\quad+\sum_{i=1}^{n} a_{n \ldots i+1}^{\dagger} a_{i-1 \ldots 1}^{\dagger} R_{0 n} \cdots R_{0 i+1} \delta_{0 i} T_{\infty 1 \ldots n}^{(n)} a_{1 \ldots n}\right\}
\end{aligned}
$$

Using

$$
\delta_{0 i} T_{\infty 1 \ldots n}^{(n)} a_{i}=T_{\infty 1 \ldots i-1,0, i+1 \ldots n}^{(n)} a_{0}
$$

and after a relabeling $j \rightarrow j-1$ for $j \geq i+1$, one gets

$$
\begin{aligned}
a_{0} \widehat{T}_{\infty}= & \sum_{n=1}^{\infty} \frac{(-1)^{n}}{n !} a_{n \ldots 1}^{\dagger} R_{0 n} \cdots R_{01} a_{0} T_{\infty 1 \ldots n}^{(n)} a_{1 \ldots n}-T_{\infty 0}^{(1)} a_{0} \\
& +\sum_{n=2}^{\infty} \frac{(-1)^{n}}{n !} \sum_{i=1}^{n} a_{n-1 \ldots 1}^{\dagger} R_{0 n-1} \cdots R_{0 i} T_{\infty 1 \ldots 0 i \ldots n-1}^{(n)} a_{1 \ldots i-1} a_{0} a_{i \ldots n-1}
\end{aligned}
$$

with, as a notation:

$i=n: R_{0 n-1} \cdots R_{0 i} \equiv 1, T_{\infty 1 \ldots 0 i \ldots n-1}^{(n)} \equiv T_{\infty 1 \ldots n-1,0}^{(n)} \quad$ and $\quad a_{1 \ldots i-1} a_{0} a_{i \ldots n-1} \equiv a_{1 \ldots n-1} a_{0}$

Rewriting

$$
a_{1 \ldots i-1} a_{0} a_{i \ldots n-1}=R_{0 i-1} \cdots R_{01} a_{0} a_{1 \ldots n-1}
$$

and relabeling $n \rightarrow n-1$ in the second summation we are led to

$$
\begin{aligned}
a_{0} \widehat{T}_{\infty}=-T_{\infty 0}^{(1)} a_{0}+ & \sum_{n=1}^{\infty} \frac{(-1)^{n}}{n !}\left\{a_{n \ldots 1}^{\dagger} R_{0 n} \cdots R_{01} a_{0} T_{\infty 1 \ldots n}^{(n)} a_{1 \ldots n}+\right. \\
& \left.-\frac{1}{n+1} \sum_{i=1}^{n+1} a_{n \ldots 1}^{\dagger} R_{0 n} \cdots R_{0 i} T_{\infty 1 \ldots n \mid i}^{(n+1)} R_{0 i-1} \cdots R_{01} a_{0} a_{1 \ldots n}\right\}
\end{aligned}
$$

that is

$$
\begin{aligned}
a_{0} \widehat{T}_{\infty}=-T_{\infty 0}^{(1)} a_{0}+ & \sum_{n=1}^{\infty} \frac{(-1)^{n}}{n !} a_{n \ldots 1}^{\dagger}\left\{\mathcal{R}_{0 n} T_{\infty 1 \ldots n}^{(n)}+\right. \\
& \left.\quad-\frac{1}{n+1} \sum_{i=1}^{n+1} \mathcal{R}_{0 n} \mathcal{R}_{0, i-1}^{-1} T_{\infty 1 \ldots n i}^{(n+1)} \mathcal{R}_{0, i-1}\right\} a_{0} a_{1 \ldots n}
\end{aligned}
$$


On the other hand, one computes

$$
R_{\infty 0} \widehat{T}_{\infty} a_{0}=\sum_{n=1}^{\infty} \frac{(-1)^{n}}{n !} a_{n \ldots 1}^{\dagger} R_{\infty 0} T_{\infty 1 \ldots n}^{(n)} \mathcal{R}_{0 n} a_{0} a_{1 \ldots n}
$$

Finally, equaling (3.5) and (3.6), we get the equations (3.1), after left-multiplication by $\mathcal{R}_{0 n}^{-1}$.

A similar calculation on $T_{\infty} a_{0}^{\dagger}=a_{0}^{\dagger} R_{\infty 0} T_{\infty}$ leads to the same equation.

Remark 3 If one defines $\mathcal{R}_{00}=\mathbb{I}$ (and $T_{\infty}^{(0)}=\mathbb{I}$ as given by $(2.10)$ ), the equation $T_{\infty 0}^{(1)}=\mathbb{I}-R_{\infty 0}$ just corresponds to $n=0$ in (3.1).

\section{Property 3.2 (Central generators of $\mathcal{A}_{R}$ )}

The only central generators of $\mathcal{A}_{R}$ are constants.

Proof: Let $c$ be a central generator of $\mathcal{A}_{R}$. Since it commutes with $a$ and $a^{\dagger}$, it also commutes with the number operator $H_{0}=\int d k a^{\dagger}(k) a(k)$ (see section 4 ). It is thus of the form

$$
c=c^{(0)}+\sum_{n=1}^{\infty} \frac{(-1)^{n}}{n !} a_{n \ldots 1}^{\dagger} c_{1 \ldots n}^{(n)} a_{1 \ldots n}
$$

Demanding $c a_{0}=a_{0} c$ leads to equations on the elements $c_{1 \ldots n}^{(n)}$. These equations are computed in the same way one computes the equations for the $T^{(n)}$ 's. Indeed, one deduces the equations on $c^{(n)}$ by formally replacing $R_{0 \infty}$ by $\mathbb{I}$ in the equations (3.1). We get the relations

$$
\begin{aligned}
& c_{1}^{(1)}=0 \\
& (n+1)\left\{c_{1 \ldots n}^{(n)}-\left(\mathcal{R}_{0, n}\right)^{-1} c_{1 \ldots n}^{(n)} \mathcal{R}_{0, n}\right\}=\sum_{i=1}^{n+1} \mathcal{R}_{0, i-1}^{-1} c_{1 \ldots n \mid i}^{(n+1)} \mathcal{R}_{0, i-1} \quad \text { for } \quad n \geq 1
\end{aligned}
$$

We prove by induction that $c^{(n)}=0$. The case $n=1$ is a direct consequence of the equations. Let us suppose that $c^{(p)}=0$ for $p \leq n$. Writing the equation (3.8) at level $n$, and using the induction, we have

$$
\sum_{i=1}^{n+1} \mathcal{R}_{0, i-1}^{-1} c_{1 \ldots n \mid i}^{(n+1)} \mathcal{R}_{0, i-1}=0
$$

Using the invariance property 2.4, we can rewrite each term of the sum has

$$
c_{1 \ldots n \mid i}^{(n+1)}=c_{1 \ldots i-1,0, i, \ldots n}^{(n+1)}=\mathcal{R}_{0, i-1} c_{01 \ldots n}^{(n+1)} \mathcal{R}_{0, i-1}^{-1}
$$

Thus, the equation is equivalent to $(n+1) c_{01 \ldots n}^{(n+1)}=0$ and the induction is proven. 
Theorem 3.3 The vertex operators $T$ is well-bred if and only if $T_{\infty 1 \ldots n}^{(n)}$ is defined by the following inductive expressions:

$$
\begin{aligned}
T_{\infty 0}^{(1)}= & \mathbb{I}-R_{\infty 0} \\
T_{\infty 01 \ldots n}^{(n+1)}= & \frac{1}{n+1} \sum_{i=0}^{n}\left(\mathcal{R}_{p_{i}}^{01 \ldots n}\right)^{-1} T_{\infty 2 \ldots i, 0, i+1, \ldots, n}^{(n)} \mathcal{R}_{p_{i}}^{01 \ldots n} \\
& -\frac{1}{(n+1) !} \sum_{\sigma \in \mathcal{S}_{n+1}}\left(\mathcal{R}_{p_{n} \circ \sigma}^{01 \ldots n}\right)^{-1} R_{\infty \sigma(0)} T_{\infty \sigma(1) \ldots \sigma(n)}^{(n)} \mathcal{R}_{p_{n} \circ \sigma}^{01 \ldots n}
\end{aligned}
$$

where $T_{\infty 2 \ldots, \ldots, i+1, . ., n}^{(n)}$ for $i=0$ stands for $T_{\infty 1 \ldots, n}^{(n)} \cdot p_{j} \in \mathcal{S}_{n+1}$ is defined by

$$
\begin{aligned}
& p_{j}:(0,1, \ldots, j-1, j, j+1, \ldots, n) \rightarrow(1,2, \ldots, j, 0, j+1, \ldots, n), 1 \leq j \leq n \\
& p_{0}=i d
\end{aligned}
$$

We remind that $R_{i j}$ stands for $R_{i j}\left(k_{i}, k_{j}\right)$.

Proof: We start with the lemma 3.1 and show that $T$ obeys the above inductive expressions. Remark that from the definition of $\mathcal{R}_{0, i}$, one has

$$
\mathcal{R}_{0, i} a_{01 \ldots n}=a_{1,2, \ldots, i, 0, i+1 \ldots n} \Rightarrow \mathcal{R}_{0, i}=\mathcal{R}_{p_{i}}^{01 \ldots n}
$$

where $p_{i}$ is defined by (3.13). We start from the equation (3.1) and work with $\mathcal{S}$ covariant matrices. Then, the right-hand side is equal to $(n+1) T_{\infty 01 \ldots n}^{(n+1)}$, while the left-hand side reads:

$$
\frac{1}{n !} \sum_{\sigma \in \mathcal{S}_{n+1}}\left(\mathcal{R}_{\sigma}^{01 \ldots n}\right)^{-1}\left\{T_{\infty \sigma(1) \ldots \sigma(n)}^{(n)}-\left(\mathcal{R}_{p_{n}}^{\sigma(0) \ldots \sigma(n)}\right)^{-1} R_{\infty \sigma(0)} T_{\infty \sigma(1) \ldots \sigma(n)}^{(n)} \mathcal{R}_{p_{n}}^{\sigma(0) \ldots \sigma(n)}\right\} \mathcal{R}_{\sigma}^{01 \ldots n}
$$

Now, we decompose $\mathcal{S}_{n+1}$ with respect to $\mathcal{S}_{n}$ : any $\sigma \in \mathcal{S}_{n+1}$ is of the form (for some $0 \leq i \leq n) \mu \circ p_{i}$ with $\mu \in \mathcal{S}_{n}$ and $p_{i}$ defined in (3.13). Using the covariance of $T^{(n)}$, one gets for the first part of the right hand side:

$$
\begin{aligned}
r h s_{1} & :=\frac{1}{n !} \sum_{\sigma \in \mathcal{S}_{n+1}}\left(\mathcal{R}_{\sigma}^{01 \ldots n}\right)^{-1} T_{\infty \sigma(1) \ldots \sigma(n)}^{(n)} \mathcal{R}_{\sigma}^{01 \ldots n} \\
& =\frac{1}{n !} \sum_{i=0}^{n} \sum_{\mu \in \mathcal{S}_{n}}\left(\mathcal{R}_{p_{i}}^{01 \ldots n}\right)^{-1}\left(\mathcal{R}_{\mu}^{p_{i}(0) p_{i}(1) \ldots p_{i}(n)}\right)^{-1} T_{\infty \mu\left(p_{i}(1)\right) \ldots \mu\left(p_{i}(n)\right)}^{(n)} \mathcal{R}_{\mu}^{p_{i}(0) p_{i}(1) \ldots p_{i}(n)} \mathcal{R}_{p_{i}}^{01 \ldots n} \\
& =\frac{1}{n !} \sum_{i=0}^{n}\left(\mathcal{R}_{p_{i}}^{01 \ldots n}\right)^{-1}\left(\sum_{\mu \in \mathcal{S}_{n}} \mathcal{T}_{\infty p_{i}(0) p_{i}(1) \ldots p_{i}(n)}^{(\mu)}\right) \mathcal{R}_{p_{i}}^{01 \ldots n}
\end{aligned}
$$

${ }^{\dagger}$ Strictly speaking, $\mu$ is still in $\mathcal{S}_{n+1}$, but it obeys $\mu(0)=0$ so that its restriction to $[1, n]$ define an element of $\mathcal{S}_{n}$. 
with $\mathcal{T}_{\infty 01 \ldots n}^{(\mu)}=\left(\mathcal{R}_{\mu}^{01 \ldots n}\right)^{-1} T_{\infty \mu(1) \ldots \mu(n)}^{(n)} \mathcal{R}_{\mu}^{01 \ldots n}$. Since $\mu(0)=0$, one has $\mathcal{R}_{\mu}^{01 \ldots n}=\mathcal{R}_{\mu}^{1 \ldots n}$. Then, using the $\mathcal{S}_{n}$-covariance of $T^{(n)}$, one gets $\mathcal{T}_{\infty 01 \ldots n}^{(\mu)}=T_{\infty 1 \ldots n}^{(n)}, \forall \mu$, so that

$$
r h s_{1}=\sum_{i=0}^{n}\left(\mathcal{R}_{p_{i}}^{01 \ldots n}\right)^{-1} T_{\infty 2 \ldots i, 0, i+1 \ldots n}^{(n)} \mathcal{R}_{p_{i}}^{01 \ldots n}
$$

Finally, to get (3.12), one remarks in the second sum of the r.h.s. that $\mathcal{R}_{p_{n}}^{\sigma(0) \ldots \sigma(n)} \mathcal{R}_{\sigma}^{01 \ldots n}=$ $\mathcal{R}_{p_{n} \circ \sigma}^{01 \ldots n}$, due to (2.20).

The same calculation (done in reverse direction) also shows that the inductive expressions obey the lemma 3.1.

Remark 4 Note that the inductive expression proves the unicity of the solution.

Remark 5 The first terms in the series (3.12) are

$$
\begin{aligned}
T_{\infty 1}^{(1)} & =\mathbb{I}-R_{\infty 1} \\
T_{\infty 12}^{(2)} & =\mathbb{I}-R_{\infty 2}+R_{\infty 2} R_{\infty 1}-R_{21} R_{\infty 1} R_{12}
\end{aligned}
$$

Corollary $3.4 \forall n \geq 0, T_{\infty 1 \ldots n}^{(n)}$ is a non-vanishing polynomial of $R$-matrices. It has the form:

$$
T_{\infty 1 \ldots n}^{(n)}=\mathbb{I}+\sum_{i=1}^{n} S_{\infty 1 \ldots n}^{(i)} \quad \text { with } \quad S_{\infty 1 \ldots n}^{(i)}=\sum_{\mu \in \mathcal{S}_{n}} m_{\mu} M_{\mu} R_{\infty \mu(1)} \cdots R_{\infty \mu(i)} M_{\mu}^{-1}
$$

where $M_{\mu}$ are products of matrices $R_{a b}$ with $1 \leq a, b \leq n$ and $m_{\mu} \in \mathbb{Z}$.

Proof: We prove the corollary by induction. The explicit expressions given above prove that it is true for $n=0,1,2$. Now, suppose (3.17) is true up to $n$. Then, the equation (3.12) shows that it is also true for $n+1$. Indeed, the two sums in (3.12) have conjugation by $R$-matrices of type $M_{\mu}$. Moreover, only the first sum contributes to $\mathbb{I}$, and effectively leads to a coefficient 1 , while the second sum increase the number of $R_{\infty a}(a=0,1, \ldots, n)$ matrices by 1 .

Remark 6 The above formula shows that $T^{(n)}$ is invertible (as a series) for all $n$.

Using the theorem 3.3 , one can show 
Property 3.5 The well-bred vertex operators $T$ of theorem 3.3 obey FRT relations:

$$
R_{12} T_{1} T_{2}=T_{2} T_{1} R_{12} \text {, i.e. } R_{12}\left(k_{1}, k_{2}\right) T_{1}\left(k_{1}\right) T_{2}\left(k_{2}\right)=T_{2}\left(k_{2}\right) T_{1}\left(k_{1}\right) R_{12}\left(k_{1}, k_{2}\right)
$$

In other words, they generate an infinite dimensional quantum group with evaluated $R$-matrix $R_{12}$. In the following, we will denote this quantum group $\mathcal{U}_{R}$.

Proof: We use the lemma 2.8 for $T: c_{12}=T_{1}^{-1} T_{2}^{-1} R_{12} T_{1} T_{2}$ is central in $\mathcal{A}_{R}$ and such that

$$
R_{12} T_{1} T_{2}=T_{2} T_{1} c_{12}
$$

$c_{12}$ being central, and due to the property 3.2, it is a constant matrix $M_{12}$. To identify the exact expression of $M_{12}$, we use the result of theorem 3.3. Looking at (3.19) as a series in the number of say $a$ operators and projecting on number 0 , we get $c_{12}=M_{12}=R_{12}$.

Remark 7 Looking at the term linear in $a$, one gets

$$
R_{12}\left(T_{13}^{(1)}+T_{23}^{(1)}-T_{13}^{(1)} \cdot T_{23}^{(1)}\right)=\left(T_{13}^{(1)}+T_{23}^{(1)}-T_{13}^{(1)} \cdot T_{13}^{(1)}\right) c_{12}
$$

Plugging into this equation the expressions of $T^{(1)}$ and $c_{12}$, one recovers the YangBaxter equation, which is indeed satisfied.

Property 3.6 Let $T$ be the well-bred vertex operator of theorem 3.3. Then, one has

$$
T^{\dagger}(k)=T(k)^{-1}
$$

Proof: From the lemma 2.7, one knows that $T^{\dagger}(k) T(k)$ is central. This implies (using property 3.2) that, $T^{\dagger}(k) T(k)$ is a constant $N \times N$ matrix $M$. Looking at the term without $a$, one concludes that $M=\mathbb{I}_{N}$.

Corollary 3.7 The expansion of $T(k)^{-1}$ as a series in a's takes the form

$$
T_{\infty}^{-1}=\mathbb{I}+\sum_{n=1}^{\infty} \frac{(-1)^{n}}{n !} a_{n \ldots 1}^{\dagger} \bar{T}_{\infty 1 \ldots n}^{(n)} a_{1 \ldots n}
$$

where $\bar{T}_{\infty 1 \ldots n}^{(n)}$ is defined by the following inductive expressions:

$$
\begin{aligned}
\bar{T}_{\infty 0}^{(1)}= & \mathbb{I}-R_{0 \infty} \\
\bar{T}_{\infty 01 \ldots n}^{(n+1)}= & \frac{1}{n+1} \sum_{i=0}^{n}\left(\mathcal{R}_{p_{i}}^{01 \ldots n}\right)^{-1} \bar{T}_{\infty 2 \ldots i, 0, i+1, \ldots, n}^{(n)} \mathcal{R}_{p_{i}}^{01 \ldots n} \\
& -\frac{1}{(n+1) !} \sum_{\sigma \in \mathcal{S}_{n+1}}\left(\mathcal{R}_{p_{n} \circ \sigma}^{01 \ldots n}\right)^{-1} \bar{T}_{\infty \sigma(1) \ldots \sigma(n)}^{(n)} R_{\sigma(0) \infty} \mathcal{R}_{p_{n} \circ \sigma}^{01 \ldots n}
\end{aligned}
$$

It obeys the corollary 3.4, with $R_{\infty \mu(i)}$ replaced by $R_{\mu(i) \infty}$ 
Proof: Simple calculation from property 2.2, theorem 3.3 and property 3.6 .

Property 3.8 The vertex operators $T$ defined in theorem 3.5 induce an isomorphism between the algebras $\mathcal{A}_{R}$ and $\mathcal{A}_{R^{-1}}$. The isomorphism is given by

$$
\tau: \begin{cases}\mathcal{A}_{R} & \rightarrow \mathcal{A}_{R^{-1}} \\ a & \mapsto \hat{a}=T^{-1} a \\ a^{\dagger} & \mapsto \hat{a}^{\dagger}=a^{\dagger} T\end{cases}
$$

Proof: We first show that $\hat{a}$ and $\hat{a}^{\dagger}$ obey the exchange relations of $\mathcal{A}_{R^{-1}}$. We remind that $R_{12}^{-1}=R_{21}$.

$$
\begin{aligned}
\hat{a}_{1} \hat{a}_{2} & =T_{1}^{-1} a_{1} T_{2}^{-1} a_{2}=T_{1}^{-1} T_{2}^{-1} R_{12} a_{1} a_{2}=R_{12} T_{2}^{-1} T_{1}^{-1} a_{1} a_{2} \\
& =R_{12} T_{2}^{-1} T_{1}^{-1} R_{21} a_{2} a_{1}=R_{12} T_{2}^{-1} a_{2} T_{1}^{-1} a_{1}=R_{12} \hat{a}_{2} \hat{a}_{1}
\end{aligned}
$$

One does a similar calculation with $\hat{a}_{1}^{\dagger} \hat{a}_{2}^{\dagger}$. In the same way, one computes

$$
\begin{aligned}
\hat{a}_{1} \hat{a}_{2}^{\dagger} & =T_{1}^{-1} a_{1} a_{2}^{\dagger} T_{2}=T_{1}^{-1} a_{2}^{\dagger} R_{12} a_{1} T_{2}+T_{1}^{-1} \delta_{12} T_{2}=T_{1}^{-1} a_{2}^{\dagger} T_{2} a_{1}+\delta_{12} \\
& =a_{2}^{\dagger} T_{1}^{-1} R_{21} T_{2} a_{1}+\delta_{12}=a_{2}^{\dagger} T_{2} R_{21} T_{1}^{-1} a_{1}+\delta_{12}=\hat{a}_{2}^{\dagger} R_{21} \hat{a}_{1}+\delta_{12}
\end{aligned}
$$

This shows that $\mathcal{A}_{R}$ is embedded into $\mathcal{A}_{R^{-1}}$. Performing the same calculation starting from $\mathcal{A}_{R^{-1}}$ proves that $\mathcal{A}_{R^{-1}}$ is embedded into $\mathcal{A}_{R}$. There is thus equality of the two algebras.

\section{Reduction to the finite dimensional case}

The above results can be applied to the case without spectral parameter. We have to start with a finite dimensional $R$-matrix obeying the Yang-Baxter equation

$$
R_{12} R_{13} R_{23}=R_{23} R_{13} R_{12}
$$

and a unitarity condition $R_{12} R_{21}=\mathbb{I}$ where, for this section only, the spectral parameters are not present. The deformed oscillators algebra is then finite dimensional, and all the properties stated above are still valid, the proofs following the same lines, omitting the integration over the spectral parameters.

Note however that the unitarity condition has still to be fulfilled, and this requirement excludes for instance the (triangular) $R$-matrix of the finite-dimensional quantum $\operatorname{group} \mathcal{U}_{q}\left(s l_{2}\right)$. 


\section{Application to integrable systems}

\section{Property 4.1 (Hierarchy associated to $\mathcal{A}_{R}$ )}

Let $H^{(n)}$ be defined by

$$
H^{(n)}=\int_{-\infty}^{\infty} d k k^{n} a^{\dagger}(k) a(k), \forall n=0,1,2, \ldots
$$

$H^{(n)}$ form an Abelian algebra, which defined a hierarchy for the algebra $\mathcal{A}_{R}$.

The evolution of the a and $a^{\dagger}$ operators under the flow $H^{(n)}$ is given by

$$
\begin{gathered}
e^{i t H^{(n)}} a(k) e^{-i t H^{(n)}}=e^{-i t k^{n}} a(k) \\
e^{i t H^{(n)}} a^{\dagger}(k) e^{-i t H^{(n)}}=e^{i t k^{n}} a^{\dagger}(k)
\end{gathered}
$$

Proof: Direct calculation. For instance:

$$
\begin{aligned}
a_{1} H^{(n)} & =a_{1} k_{2}^{n} a_{2}^{\dagger} a_{2}=k_{2}^{n}\left(a_{2}^{\dagger} R_{12} a_{1} a_{2}+\delta_{12} a_{2}\right)=k_{2}^{n} a_{2}^{\dagger} R_{12} R_{21} a_{2} a_{1}+k_{1}^{n} a_{1} \\
& =H^{(n)} a_{1}+k_{1}^{n} a_{1}
\end{aligned}
$$

and thus $\left[H^{(n)}, a_{1}\right]=-k_{1}^{n} a_{1}$.

Property 4.2 Any well-bred operator $L$ is an integral of motion for the hierarchy:

$$
\left[L, H^{(n)}\right]=0, \forall n=0,1,2, \ldots
$$

In particular, it is the case of the well-bred vertex operators defined in theorem 3.马, and the quantum group $\mathcal{U}_{R}$ generates an infinite dimensional symmetry algebra for the hierarchy.

Proof: $L_{1} H_{2}^{(m)}=k_{2}^{m} L_{1} a_{2}^{\dagger} a_{2}=k_{2}^{m} a_{2}^{\dagger} R_{12} L_{1} a_{2}=k_{2}^{m} a_{2}^{\dagger} R_{12} R_{21} a_{2} L_{1}=H_{2}^{(m)} L_{1}$

Remark 8 From the example of section 6.1 (see below), and which was studied in [4, 2], we conjecture that to each $\mathcal{A}_{R}$-hierarchy corresponds an integrable system already studied in the literature. The $a^{\dagger}$ operators in the Fock space representation, in this context, will correspond to asymptotic states of the system. The correlation functions of the system would then be computed using the $a^{\dagger}$ operators. 


\section{$5 \quad$ Fock space and evaluation representations}

Associated to the deformed oscillator algebra $\mathcal{A}_{R}$ comes the notion of Fock space:

Definition 5.1 The Fock space $\mathcal{F}_{R}$ of the $\mathcal{A}_{R}$ algebra is the module generated by the vacuum $\Omega$ such that

$$
a_{i}(k) \Omega=0 \quad \forall i=1, \ldots, N \forall k
$$

Now, since one has constructed a quantum group from the $\mathcal{A}_{R}$ algebra, it is natural to look at the representations induced by the Fock space:

Property 5.2 The Fock space $\mathcal{F}_{R}$ decomposes under the action of the Hamiltonians $H^{(n)}$ into an infinite sum of tensor product of evaluation representations of $\mathcal{U}_{R}$ :

$$
\mathcal{F}_{R}=\oplus_{n=0}^{\infty} \int d k_{1} \cdots d k_{n} \theta\left(k_{1} \leq k_{2} \leq \ldots \leq k_{n}\right) \mathcal{V}_{n}\left(k_{1}, k_{2}, \ldots, k_{n}\right)
$$

where $\theta\left(k_{1} \leq k_{2} \leq \ldots \leq k_{n}\right)$ indicates that the spectral parameters are ordered.

In particular, the representations $\mathcal{V}_{n}\left(k_{1}, \ldots, k_{n}\right)$ are of dimension $N^{n}$, and $T$ acts in these spaces by right-multiplication by $R$.

Proof: Since the Hamiltonians $H^{(n)}$ form a commuting subalgebra of $\mathcal{A}_{R}$, we can consider them as a Cartan subalgebra, and decompose $\mathcal{F}_{R}$ into Cartan-eigenspaces $\mathcal{V}_{n}\left(h_{1}, h_{2}, \ldots\right)$, where $n$ denotes the eigenvalue under $H^{(0)}$ (which turn to be still the particle number although we are in the deformed case) and $h_{p}$ is the eigenvalue of $H^{(p)}$ $(p>0)$. Now, since $\mathcal{U}_{R}$ commutes with these Hamiltonians, the eigenspaces are stable under the action of $\mathcal{U}_{R}$ and thus are representations of $\mathcal{U}_{R}$.

The vectors in $\mathcal{F}_{R}$ are linear combinations of monomials $a_{\alpha_{1}}^{\dagger}\left(k_{1}\right) \cdots a_{\alpha_{m}}^{\dagger}\left(k_{m}\right) \Omega, \forall m$. On the eigenspace $\mathcal{V}_{n}\left(h_{0}, h_{1}, h_{2}, \ldots\right)$, one must consider only monomials with $m=n$ : this provides only a finite number of terms, and the eigenspace is of finite dimension. Moreover, the eigenvalues under the $H^{(n)}$ being fixed, one has equations

$$
h_{1}=\sum_{i=1}^{n} k_{i} ; h_{2}=\sum_{i=1}^{n} k_{i}^{2} ; \ldots ; h_{n}=\sum_{i=1}^{n} k_{i}^{n}
$$

which completely fixes the values of $k_{1}, \ldots, k_{n}$ (up to a permutation) and also of $h_{p}=$ $\sum_{i=1}^{n} k_{i}^{p}, p>n$. Thus, we can replace the labeling $h_{1}, h_{2}, \ldots$ by $k_{1}, \ldots, k_{n}$, whence the notation $\mathcal{V}_{n}\left(k_{1}, \ldots, k_{n}\right)$ for the representations of $\mathcal{U}_{R}$. Finally, the exchange relations among the $a^{\dagger}$ 's allow to reorder them in such a way that the spectral parameters are in increasing order.

Because it is a vertex operator, the action of $T$ on $\Omega$ is trivial, and since it is wellbred, its action on other states is a multiplication by $R$. 


\section{Remark 9 (Hopf structure of $\mathcal{U}_{R}$ )}

Although one cannot obtain the Hopf structure of $\mathcal{U}_{R}$ starting from $\mathcal{A}_{R}$, one can infer it from the present construction in the following way.

The "first" eigenspaces are

$$
\begin{aligned}
\mathcal{V}_{0}(0) & =\mathbb{C} \Omega \\
\mathcal{V}_{1}(k) & =\operatorname{Span}\left(a_{i}^{\dagger}(k) \Omega, i=1, \ldots, N\right) \\
\mathcal{V}_{2}\left(k_{1}, k_{2}\right) & =\operatorname{Span}\left(a_{j}^{\dagger}\left(k_{2}\right) a_{i}^{\dagger}\left(k_{1}\right) \Omega, k_{1} \leq k_{2}, i, j=1, \ldots, N\right)
\end{aligned}
$$

Looking at the action of the well-bred vertex operators $T$ on these spaces, one gets

$$
T \Omega=\Omega \quad ; \quad T_{1} a_{2}^{\dagger} \Omega=a_{2}^{\dagger} R_{12} \Omega \quad ; \quad T_{1} a_{2}^{\dagger} a_{3}^{\dagger} \Omega=a_{2}^{\dagger} R_{12} a_{3}^{\dagger} R_{13} \Omega
$$

Interpreting $\mathcal{V}_{2}\left(k_{1}, k_{2}\right)$ as the tensor product $\mathcal{V}_{1}\left(k_{1}\right) \otimes \mathcal{V}_{1}\left(k_{2}\right)$,

$$
a_{2}^{\dagger} a_{3}^{\dagger} \Omega \sim a_{2}^{\dagger} \Omega \otimes a_{3}^{\dagger} \Omega
$$

we getf

$$
T_{1} a_{2}^{\dagger} a_{3}^{\dagger} \Omega=a_{2}^{\dagger} R_{12} a_{3}^{\dagger} R_{13} \Omega \sim a_{2}^{\dagger} R_{12} \Omega \otimes a_{3}^{\dagger} R_{13} \Omega=\left(T_{1} \otimes T_{1}\right)\left(a_{2}^{\dagger} \Omega \otimes a_{3}^{\dagger} \Omega\right)
$$

Thus, we are naturally led to the coproduct formula

$$
\Delta(T)=T \otimes T
$$

which is the right one for $\mathcal{U}_{R}$.

Remark 10 Note also that, due to the finite number of $a$ operators in the states of $\mathcal{V}_{m}$, the vertex operators truncate at level $m$, and turn to be polynomials in $a, a^{\dagger}$ in these representations.

\section{$6 \quad$ Examples}

We treat here two examples: one associated with an additive spectral parameter, and the second one to a multiplicative spectral parameter.

\footnotetext{
$\mathcal{A}_{R}$.

${ }^{\ddagger}$ Be careful that the indices 1, 2, 3 refer to the auxiliary spaces while the tensor product refers to
} 


\subsection{The nonlinear Schrödinger equation}

The nonlinear Schrödinger equation in 1+1 dimensions (NLS) has been widely studied. We look at it in the QISM approach ( for a review, see for instance [5] and ref. therein).

It has already been shown [4, 2] that all the informations on the hierarchy associated to NLS can be reconstructed starting from the algebra $\mathcal{A}_{R}$, where $R$ is the $R$-matrix of the Yangian $Y(N)$ based on $g l(N)$ :

$$
R(k)=\frac{1}{k+i g}\left(k \mathbb{I}_{N} \otimes \mathbb{I}_{N}+i g P_{12}\right) \quad, \quad P_{12}=\sum_{i, j=1}^{N} E_{i j} \otimes E_{j i}
$$

This $R$-matrix obey an additive Yang-Baxter equation

$$
R_{12}\left(k_{1}-k_{2}\right) R_{13}\left(k_{1}-k_{3}\right) R_{23}\left(k_{2}-k_{3}\right)=R_{23}\left(k_{2}-k_{3}\right) R_{13}\left(k_{1}-k_{3}\right) R_{12}\left(k_{1}-k_{2}\right)
$$

and one shows, using $P^{2}=\mathbb{I}$, that $R_{12}(k) R_{21}(-k)=\mathbb{I}$. Thus, the properties stated above apply.

In fact, it is well-known that the canonical field $\Phi$ obeying the (quantum) NLS:

$$
\left(i \partial_{t}+\partial_{x}^{2}\right) \Phi(x, t)=2 g: \Phi(x, t) \bar{\Phi}(x, t) \Phi(x, t): \quad \text { with } \quad \Phi(x, t)=\left(\begin{array}{c}
\varphi_{1}(x, t) \\
\vdots \\
\varphi_{n}(x, t)
\end{array}\right)
$$

can be reconstructed from $\mathcal{A}_{R}$ [4]. The Hamiltonian is then exactly $H^{(2)}$, and the Yangian $Y(N)$ is a symmetry of the hierarchy [3, 2]. The operators $a^{\dagger}$ correspond to asymptotic states in the Fock space $\mathcal{F}$.

The generators $Q_{0}^{a}$ and $Q_{1}^{a}$ of $Y(N)$ in its Drinfeld presentation were built in term of $\mathcal{A}_{R}$ in [2] (see also [3 for the $g l_{2}$ case). The present approach is an alternative construction of $Y(N)$ in the FRT presentation. It has the advantage to give an explicit construction for all the generators of the Yangian, and also to give the action of these generators (i.e. of the integrals of motion) on the $a$ and $a^{\dagger}$ operators (i.e. the asymptotic states of the system).

\subsection{The quantum group $\mathcal{U}_{q}\left(\widehat{g l_{2}}\right)$}

We take here the evaluated $R$-matrix of the centerless affine $g l_{2}$ quantum algebra. Following the usual notation, the spectral parameter is denoted $z$. The $R$-matrix reads:

$$
R(z)=\left(\begin{array}{cccc}
1 & 0 & 0 & 0 \\
0 & \frac{q\left(1-z^{2}\right)}{1-q^{2} z^{2}} & \frac{z\left(1-q^{2}\right)}{1-q^{2} z^{2}} & 0 \\
0 & \frac{z\left(1-q^{2}\right)}{1-q^{2} z^{2}} & \frac{q\left(1-z^{2}\right)}{1-q^{2} z^{2}} & 0 \\
0 & 0 & 0 & 1
\end{array}\right)
$$


It is defined here up to a normalization factor $\rho$ such that the unitarity condition $R_{12}\left(z_{1} / z_{2}\right) R_{21}\left(z_{2} / z_{1}\right)=1$ is preserved, i.e.

$$
\rho(z) \rho\left(\frac{1}{z}\right)=1 .
$$

The $R$-matrix obeys a multiplicative Yang-Baxter equation:

$$
R_{12}\left(z_{1} / z_{2}\right) R_{13}\left(z_{1} / z_{3}\right) R_{23}\left(z_{2} / z_{3}\right)=R_{23}\left(z_{2} / z_{3}\right) R_{13}\left(z_{1} / z_{3}\right) R_{12}\left(z_{1} / z_{2}\right)
$$

and once again, one can apply the above properties. Note however that we are forced to take a vanishing central charge, so that the algebra $\mathcal{U}_{q}\left(\widehat{g l}_{2}\right)$ is defined by the relation

$$
R_{12}\left(z_{1} / z_{2}\right) T_{1}\left(z_{1}\right) T_{2}\left(z_{2}\right)=T_{2}\left(z_{2}\right) T_{1}\left(z_{1}\right) R_{12}\left(z_{1} / z_{2}\right)
$$

The Hamiltonian $H^{(2)}$ should correspond to the Hamiltonian of Sine-Gordon model.

\subsection{The elliptic quantum group $\mathcal{A}_{q, p}\left(\widehat{g l_{2}}\right)$}

The elliptic quantum group $\mathcal{A}_{q, p}\left(\widehat{g l}_{2}\right)_{c}$ has defining relations

$$
R_{12}\left(z_{1} / z_{2} ; q, p\right) T_{1}\left(z_{1}\right) T_{2}\left(z_{2}\right)=T_{2}\left(z_{2}\right) T_{1}\left(z_{1}\right) R_{12}^{*}\left(z_{1} / z_{2} ; q, p\right)
$$

where $R_{12}^{*}(z ; q, p)=R_{12}\left(z ; q, p q^{-2 c}\right)$. Note that $R_{12}$ obeys the unitarity condition. Thus, in the centerless case, one has $R^{*}=R$, and the above procedure can be applied. One will start with the evaluated $R$-matrix of $\mathcal{A}_{q, p}\left(\widehat{g l_{2}}\right)_{c=0}$ and construct the corresponding ZF algebra.

In this way, one gets a well-bred vertex operator that realizes $\mathcal{A}_{q, p}\left(\widehat{g l_{2}}\right)_{c=0}$, and this latter algebra is a symmetry of the hierarchy associated to the ZF algebra. In particular, the Hamiltonian $H^{(2)}$ should be related to the XYZ model, and in this framework, we naturally gets $\mathcal{A}_{q, p}\left(\widehat{g l_{2}}\right)_{c=0}$ as a symmetry of this model.

\section{Conclusion and perspectives}

Starting with any $R$-matrix with spectral parameter, obeying the Yang-Baxter equation and a unitarity condition, we have constructed the corresponding quantum group $\mathcal{U}_{R}$ in term of a deformed oscillators algebra $\mathcal{A}_{R}$. The realization we present is an infinite series, the expansion being given in the number of creation operators. Up to a normalization constant, the construction is unique. These "well-bred vertex operators" act naturally on $\mathcal{A}_{R}$. As a consequence, they are integrals of motion of the integrable hierarchy naturally associated to $\mathcal{A}_{R}$.

Taking as an example the $R$-matrix of $Y(N)$, the Yangian based on $g l(N)$, we recover by this construction the nonlinear Schrödinger equation and its $Y(N)$ symmetry. It is thus very natural to believe that the other integrable systems known in the literature can be treated with the present approach. 
Of course, the comparison between the vertex operators constructed in this paper, and the vertex operators of quantum affine algebras known in the literature (e.g. [6]) has to be done. Note however that our construction can be done for any infinite quantum group, provided its evaluated $R$-matrix obeys the unitarity condition.

As a generalization, it is natural to ask whether such an approach can be extended to the case of (elliptic) quantum groups with non-vanishing central charge: this seems to be very much the case [7]. If such a generalization can be done, it would then be possible to look at (off-shell) correlation functions for the underlying integrable systems. Moreover, this could give a pertinent insight in the research of vertex operators, as they are looked for when starting with the canonical fields of the integrable system [8].

\section{Acknowledgments}

I would like to thank D. Arnaudon and L. Frappat for fruitful remarks on the center of ZF algebras.

I am grateful to the referee for pertinent remarks, specially pointing out a mistake in the first version of lemma 3.1 and theorem 3.3 .

\section{References}

[1] A. B. Zamolodchikov and A. B. Zamolodchikov, Ann. Phys. 120 (1979) 253;

L. D. Faddeev, Soviet Scientific Reviews Sect. C 1 (1980) 107.

[2] M. Mintchev, E. Ragoucy, P. Sorba and Ph. Zaugg, J. Phys. A32 (1999) 5885.

[3] S. Murakami and M. Wadati, J. Phys. A29 (1996) 7903.

[4] E. Sklyanin, L. D. Faddeev, Sov. Phys. Dokl. 23 (1978) 902;

E. Sklyanin, Sov. Phys. Dokl. 24 (1979) 107;

H.B. Tacker, D. Wilkinson, Phys. Rev. D19 (1979) 3660;

D.B. Creamer, H.B. Tacker, D. Wilkinson, Phys. Rev. D21 (1980) 1523;

J. Honerkamp, P. Weber, A. Wiesler, Nucl. Phys. B152 (1979) 266;

B. Davies, J. Phys. A14 (1981) 2631.

[5] E. Gutkin, Phys. Rep. 167 (1988) 1.

[6] I. Frenkel and N. Jing, Proc. Nat. Acad. Sci. USA 85 (1988) 9373;

Bai-Qi Jin, Shan-You Zhou, Vertex Operator of $U_{q}\left(\widehat{B_{l}}\right)$ for Level One, q-alg/9512005.

[7] D. Arnaudon, L. Frappat and E. Ragoucy, work in progress. 
[8] Y. Hara, M. Jimbo, H. Konno, S. Odake, J. Shiraishi, On Lepowsky-Wilson's Zalgebra, mathQA/0005203;

Y.Hara, M.Jimbo, H.Konno, S.Odake, J.Shiraishi, Free Field Approach to the Dilute $A_{L}$ Models, J. Math. Phys. 40 (1999) 3791, math.QA/9902150;

M. Jimbo, H. Konno, S. Odake, J. Shiraishi, Comm. Math. Phys. 199 (1999) 605, math.QA/9802002. 